\title{
Assessment of Diagnostic Accuracy of Knee MRI in Detecting Medial Parapatellar Plicae versus Knee Arthroscopic Findings
}

\author{
Muhyeddine Al-Taki ${ }^{\star *}$, Hamdi Sukkarieh ${ }^{1}$, Nabil Khoury ${ }^{2}$, Hani Tamim ${ }^{3}$, Ali H Artail ${ }^{1}$, Joel Wazzan ${ }^{3}$ and Mohammad Nassereddine ${ }^{1}$ \\ ${ }^{1}$ Department of Surgery, Division of Orthopaedic Surgery, American University of Beirut Medical Center, Beirut, Lebanon \\ ${ }^{2}$ Division of Diagnostic Radiology, American University of Beirut Medical Center, Beirut, Lebanon \\ ${ }^{3}$ Department of Internal Medicine, Clinical Research Unit, American University of Beirut Medical Center, Beirut, Lebanon
}

"Corresponding author: Muhyeddine Al-Taki, Division of Orthopaedic Surgery, Department of Surgery, American University of Beirut Medical Center, Beirut, Lebanon, Tel: + 961 (1) 350000; Fax: +961 (1) 363291; E-mail: maltaki@aub.edu.lb

Received Date: June 29, 2018; Accepted Date: July 09, 2018; Published Date: July 14, 2018

Copyright: $\odot 2018$ Al-Taki M, et al. This is an open-access article distributed under the terms of the Creative Commons Attribution License, which permits unrestricted use, distribution, and reproduction in any medium, provided the original author and source are credited.

\begin{abstract}
Background: Medial Parapatellar synovial plicae is a common cause of knee pain with a clinical presentation similar to meniscal tear but with a different pathophysiology. Plicae, an extension of the protective synovial capsule of the knee, are originally remnants of embryological folds inside the knee joint that fail to regress with time. Although benign in nature, plicae, when thickened, can impinge on the medial femoral condyle leading to pain. Magnetic resonance imaging is the non-invasive test of choice for detecting Parapatellar plicae.
\end{abstract}

Objective: The objective of this study was to assess the specificity, sensitivity and diagnostic accuracy of MRI in detecting medial parapatellar synovial plicae as compared to knee arthroscopy findings.

Methods: A retrospective review was conducted at the American University of Beirut Medical Center. Patients who underwent knee arthroscopy and were between 18 and 75 years of age were included. Data on age, gender, knee arthroscopy findings and preoperative MRI findings were abstracted. The diagnostic capability of MRI was assessed by calculating the sensitivity, specificity, positive predictive value, and negative predictive value.

Results: 29 MRIs were read as negative and 52 were positive for the presence of medial Parapatellar plica. Out of the 23 negative MRIs, $14(60.8 \%)$ had plicae as demonstrated during arthroscopy and $9(39.2 \%)$ had no plica as confirmed during arthroscopy. Whereas $34.4 \%$ (20 out of 58 ) patients with arthroscopic demonstrated plicae had negative MRI readings, and $63.6 \%$ (38 out of 58 ) of patients with plicae demonstrated on arthroscopy had actual positive findings on MRI.

Conclusion: MRI accuracy in detecting plica is insufficient compared to knee arthroscopic findings. This take home message might be a helpful tool for insurance company to acknowledge the limitation of MRI in diagnosing plica syndrome due to the significant probability of misdiagnosis.

Keywords: Knee pain; Parapatellar plicae

\section{Introduction}

Knee pain is a major cause of disability and one of the main orthopaedic complaints seen in the general population with a prevalence of $14 \%$ to $47 \%$ [1-3]. Knee pain has many etiologies ranging from arthritis to meniscal and ligamentous injuries. Synovial plicae another cause of knee pain, has a clinical presentation similar to meniscal injury. Symptoms include but not limited to popping, catching, snapping, crepitation, and/or knee swelling. Many synovial plicae are found in the knee joint and they include suprapatellar, lateral patellar, infrapatellar, and medial patellar plica.

Compared to rest of the plica the medial Parapatellar plicae are the most common cause of knee pain and the most studied followed by suprapatellar, infrapatellar and then lateral plica. The incidence of medial plicae varies between studies. Sakakibara [4] reported a rate of $55 \%$ and cadaveric study showed an incidence of $34 \%$. The prevalence was even found to be $95 \%$ in patients suffering from chronic knee pain in a cohort of more than 40-years-old individuals (Figure 1) [5].

Medial Parapatellar plica syndrome has been implicated in osteoarthritis of the knee and has been described as 'a painful impairment of knee function' that can only be explained by the presence of a thickened, hypertrophied plica [6]. Medial Parapatellar plicae may cause irritation and pain secondary to impingement on the medial femoral condyle, presenting either medial to the patella, above the joint line, or as a palpable cord upon palpation. These signs are pathognomonic to the condition [6].

Synovial plicae are embryologic septum remnants that have not fully obliterated and range in shape from a ridge to a more distinct cord [7]. Alternatively, the medial Parapatellar plica has also been described as a remnant of mesenchymal tissue from the medial aspect of the patella femoral area (Figure 2) [8-13]. 


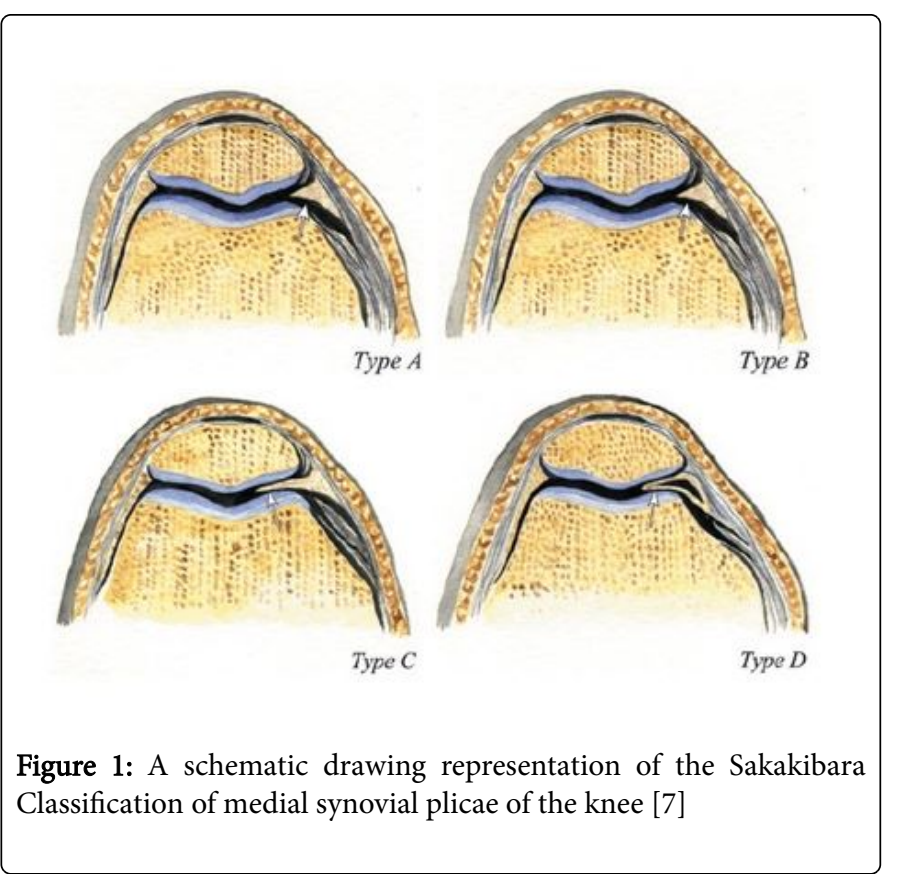

On imaging and on arthroscopic visualization, medial Parapatellar plicae appear as thin structures that originate suprapatellarly and cross obliquely and inferiorly towards the infra patellar fat pad. On arthroscopy, one can also appreciate their soft appearance and flexible nature. MRI is the non-invasive test of choice for detecting plica and they are typically seen as low-intensity signal structures delineated by high-intensity joint fluid in the joint space on a fat suppressed T2 weighted image [7]. Medial Parapatellar plicae are classified into 4 types, A through D as described by Sakakibara (Figure 3) [4]. They vary along a spectrum with type A being the smallest, thin, and cordlike to type D being the biggest with a fenestrated shelf like protrusion. Medial plicae are not the most common type of plica; however, they tend to be the most problematic, particularly types $\mathrm{C}$ and $\mathrm{D}$. These types may become entrapped between the medial femoral condyle and the patella when the knee is in extension, and impinge on the articular cartilage of the medial aspect of the patella when in flexion [4].Chronic irritation of the plica by inflammation of the surrounding synovial tissue can lead to fibrosis, making the plica less elastic and more cord-like. Although MRI is helpful in diagnosis, arthroscopy remains the gold standard for diagnosis and treatment. Treatment consists initially of conservative measures and if that fails arthroscopic excision is indicated.

Figure 1: A schematic drawing representation of the Sakakibara Classification of medial synovial plicae of the knee [7]

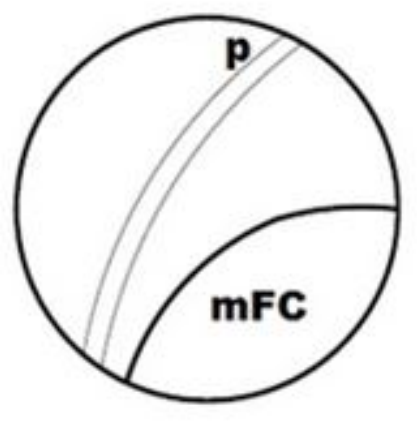

Upon knee extension

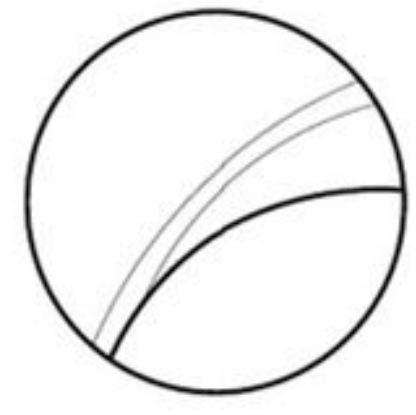

Upon $15-30^{\circ}$ flexion

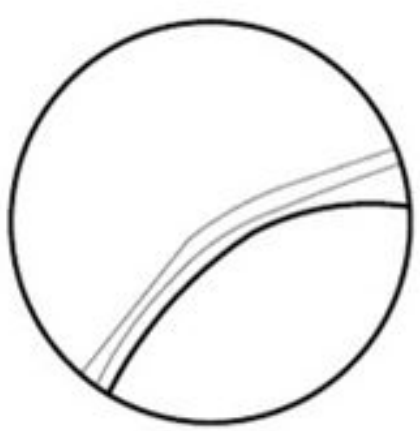

Upon $30-45^{\circ}$ flexion

Figure 2: Illustrating how a plica contacts the medial femoral condyle during normal knee range of motion, from left to right: extension, 30 degrees of flexion, 45 degrees of flexion. mFC: medial femoral condyle, P: plica.

Controversy surrounds the cost-effectiveness of the diagnosis via arthroscopy, and tends to be challenged by third-party payers. These third-party payers usually rely on MRI results to decide whether or not to issue coverage. However, the value of MRI is controversial in the detection and diagnosis of medial Parapatellar plicae. In this study, we sought to determine the sensitivity and specificity of MRI as a diagnostic tool for medial Parapatellar plicae and to compare its diagnostic utility to that of the arthroscope.

\section{Methods}

A retrospective study of knee arthroscopy cases at the American University of Beirut Medical Center between July 2010 and September 2013 was carried out. Patients who underwent knee arthroscopy and were between 18 and 75 years of age were included. This was true irrespective of the underlying pathology as recorded by the primary investigator. Those patients who had prior knee surgery, history of advanced knee osteoarthritis, inflammatory conditions affecting the knee, knee infection, knee trauma with hemarthrosis, or whose MRI was not available were excluded from the study. The approval of the Institutional Review Board at the American University of Beirut was obtained prior to initiation of the study.

The medical charts of the study subjects or these patients were reviewed and demographic variables, relevant information, and the presence of medial Parapatellar synovial plica, as seen intra operatively, were recorded. The arthroscopic finding of a medial Parapatellar plica was considered to be a definite diagnosis of Plica syndrome. Preoperative knee MRI of these patients was also reviewed. The MRIs were also read by a musculoskeletal radiologist for who was blinded to the prior initial report that was issued by the original radiologist and 
Citation: $\quad$ Al-Taki M, Sukkarieh H, Khoury N, Tamim H, Artail AH, et al. (2018) Assessment of Diagnostic Accuracy of Knee MRI in Detecting Medial Parapatellar Plicae versus Knee Arthroscopic Findings. J Surg Anesth 2: 116.

Page 3 of 5

was also blinded to the arthroscopic findings. The first review was discarded and the new reading included data on the presence of a medial Parapatellar synovial plica, its thickness, classification of severity, and joint effusion evaluation. The presence or absence of plica on MRI was the primary endpoint and was compared to the arthroscopic finding.
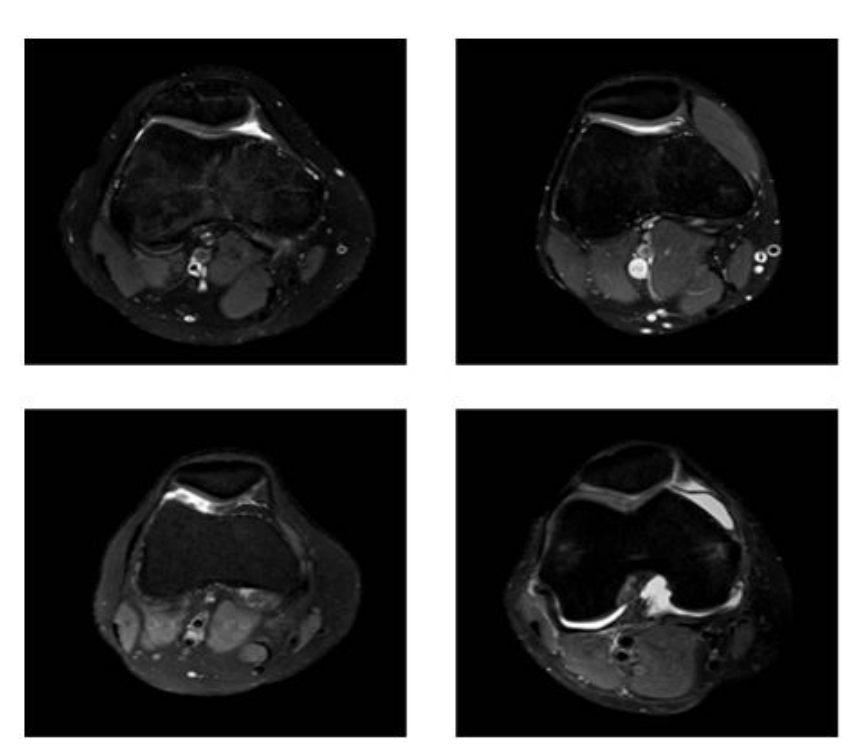

Figure 3: MRI T2W axial images showing the different types of medial Parapatellar plica as classified by Sakakibara Clockwise from top left: types A, B, C, and D.

\section{Statistical analyses}

The data was entered and analysed using SPSS 20 (SPSS Inc. Chicago, IL). Descriptive analyses of all variables were carried out and reported as count and percept for the categorical variables and as mean and standard deviation (SD) for the continuous variables. The difference between those with positive arthroscopic finding and those negative was assessed using student's t-test and Chi-square test, as appropriate. The sensitivity, specificity, predictive values, likelihood ratios, and confidence intervals were calculated to assess the efficiency of the MRI diagnostic capability as compared to the arthroscopic findings. Moreover, the $95 \%$ confidence interval (CI) for those measures were also calculated. The p-value $>0.005$ was used to indicate statistical significance.

\section{Results}

A total of 81 cases were included in this study. The average age of patients with arthroscopically demonstrated plicae was found to be $43.48 \pm 16.40$ years and for those without plicae by arthroscopy was $41.48 \pm 17.41$ years (p-value 0.61 ). The majority of patients in both groups were males, where $57.7 \%$ of patients with arthroscopically demonstrated plicae and $82.8 \%$ without plicae were males.

We found that initially 29 MRIs were read as negative and 52 were positive for the presence of medial Parapatellar plica. Out of the 23 negative MRIs, $14(60.8 \%)$ had plicae as demonstrated during arthroscopy and $9(39.2 \%)$ had no plica as confirmed during arthroscopy. Whereas $34.4 \%$ (20 out of 58) patients with arthroscopic demonstrated plicae had negative MRI readings, and 63.6\% (38 out of 58) of patients with plicae demonstrated on arthroscopy had actual positive findings on MRI (Table 1).

\begin{tabular}{|l|l|l|l|}
\hline \multirow{2}{*}{$\begin{array}{l}\text { Patient } \\
\text { Characteristics }\end{array}$} & \multicolumn{3}{|l|}{ Arthroscopy Finding (Presence of Plicae) } \\
\cline { 2 - 4 } & Negative (n=29) & Positive (n=52) & p-value \\
\hline Age, mean (SD) & $41.48 \pm 17.41$ & $43.48 \pm 16.40$ & 0.61 \\
\hline$\leq 40$ & $12(41.4 \%)$ & $25(48.1 \%)$ & 0.34 \\
\hline$>40$ & $17(58.6 \%)$ & $27(51.9 \%)$ & \\
\hline Gender, Male & $24(82.8 \%)$ & $30(57.7 \%)$ & 0.02 \\
\hline
\end{tabular}

Table 1: Patient demographics

Furthermore, plica thickness by MRI, age, and the presence of joint effusion by MRI had no significant association with the arthroscopic presence of plica. The thickness of the plica as estimated by MRI was found to be $0.92 \pm 0.28 \mathrm{~mm}$ for those who were found to not have plicae on arthroscopy, and $0.96 \pm 0.33 \mathrm{~mm}$ for those with arthroscopically demonstrated plicae ( $\mathrm{p}$ value 0.67 ). Joint effusion was absent in $6.9 \%$ ( 2 out of 23 patients without plicae by scope) patients, and was present in $93.1 \%$ (27 out of 29 ) in those without plicae by arthroscope. Out of the 52 patients with plicae by arthroscopy, 49 (94.2\%) patients had joint effusions (P value 0.22) (Table 2). Additionally, we found that the majority of arthroscopically confirmed plicae were male, with the false negatives being $82.8 \%$ male and true positives being $57.7 \%$ male. Moreover, there appears to be a statistically significant difference $(p=0.02)$ that shows a correlation between males and plicae found on arthroscopy with a negative MRI reading.

\begin{tabular}{|l|l|l|l|}
\hline \multicolumn{2}{|l|}{ Arthroscopy Finding (Presence of Plicae) } \\
\hline MRI findings & Negative (n=29) & Positive (n=52) & \multicolumn{2}{|l|}{ p-value } \\
\hline Sakakibara classification & \multicolumn{2}{|l|}{} \\
\hline Negative finding & $9(31.0 \%)$ & $14(26.9 \%)$ & \multirow{2}{*}{0.58} \\
\hline A & $8(27.6 \%)$ & $14(26.9 \%)$ & \\
\hline B & $9(31.0 \%)$ & $22(42.3 \%)$ & \\
\hline C & $3(10.3 \%)$ & $2(3.8 \%)$ & 0.67 \\
\hline Plicae Thickness $(\mathbf{m m})$ & $0.92 \pm 0.28$ & $0.96 \pm 0.33$ & \\
\hline
\end{tabular}




\begin{tabular}{|c|c|c|c|}
\hline \multicolumn{4}{|c|}{ Joint effusion } \\
\hline No & $2(6.9 \%)$ & $3(5.8 \%)$ & \multirow{4}{*}{0.22} \\
\hline Small & $19(65.5 \%)$ & $27(51.9 \%)$ & \\
\hline Medium & $6(20.7 \%)$ & $21(40.4 \%)$ & \\
\hline Large & $2(6.9 \%)$ & $1(1.9 \%)$ & \\
\hline
\end{tabular}

Table 2: The frequency of MRI findings in patients who tested positive or negative in arthroscopy

Outcome measures showed the sensitivity of MRI to be $73 \%$ (38 out of 52), and the specificity was found to be $31 \%$ ( 9 out of 29). The positive predictive value (PPV) was $65 \%$ and the negative predictive value (NPV) was found to be $39 \%$.Positive likelihood ratio was found to be 1.05 (95\% CI: 0.79-1.42) and negative likelihood ratio 0.87 (95\% CI: 0.48-1.58). When broken down further by dividing into above and below 40 years of age, specificity was $41.7 \%$ and sensitivity $88 \%$ for those 40 years of age or less. For those older than 40 years specificity was $23.5 \%$ and sensitivity $59.3 \%$. Similarly when stratified by age, for those 40 -years-old or younger PPV was $76 \%$ and NPV 63\% while for those older than 40 years the PPV was 55\% and NPV 27\%. Positive likelihood ratio for those 40 years of age or younger were found to be 1.51 (95\% CI: 0.92-2.49) and negative likelihood ratio 0.29 (95\% CI: 0.08-1.01), and for those older than the age of 40 positive likelihood ratio 0.77 (95\% CI: 0.51-1.17) and negative likelihood ratio 1.73 (95\% CI: 0.66-4.57) (Table 3).

\begin{tabular}{|l|l|l|l|}
\hline & Overall & $\mathbf{S} \mathbf{4 0}$ years of age & $>\mathbf{4 0}$ years of age \\
\hline Sensitivity & $0.73(0.59-0.84)$ & $0.88(0.68-0.97)$ & $0.59(0.40-0.77)$ \\
\hline Specificity & $0.31(0.16-0.51)$ & $0.42(0.17-0.71)$ & $0.24(0.08-0.50)$ \\
\hline Positive Predictive Value & $0.65(0.52-0.77)$ & $0.76(0.56-0.89)$ & $0.55(0.36-0.73)$ \\
\hline Negative Predictive Value & $0.39(0.20-0.61)$ & $0.63(0.26-0.90)$ & $0.27(0.09-0.55)$ \\
\hline Positive Likelihood Ratio & $1.05(0.79-1.42)$ & $1.51(0.92-2.49)$ & $0.77(0.51-1.17)$ \\
\hline Negative Likelihood Ratio & $0.87(0.48-1.58)$ & $0.29(0.08-1.01)$ & $1.73(0.66-4.57)$ \\
\hline
\end{tabular}

Table 3: Sensitivity, specificity, and predictive values and 95\% CI (between parentheses) of MRI for medial Parapatellar syndrome both for all age groups, for patients 40 -years-old or less and for those above 40 years of age

\section{Discussion}

Plicae are thickenings of the synovial folds that can cause knee symptoms, including pain, that mimic many entities including meniscal tears, osteoarthritis and other inflammatory conditions. Previously, the clinical significance of the medial Parapatellar plica was debatable, when other types of plicae, although more frequent, have been known to have minimal clinical significance. Currently, medial Parapatellar plicae are well known to cause knee pain [11], and have even been implicated in the pathogenesis of medial compartment knee osteoarthritis as demonstrated in a previously published histomorphologic study $[13,14]$. However, diagnosing plicae as the root cause of pain remains problematic. The definitive method for establishing the presence of a plica is by arthroscopy, an invasive procedure [10]. MRI is the non-invasive technique being used by clinicians and healthcare coverage companies as the method of choice for demonstrating the presence of a plica. Coverage of treatment by third-party payers often hinges on MRI-demonstrated presence of plicae or other pathologies in the knee. The use of MRI for diagnosis is still controversial due to its sensitivity and specificity in diagnosing medial Parapatellar plica syndrome. In our current study, we elaborated on the sensitivity and specificity of MRI in detecting the presence of medial Parapatellar plicae with arthroscopic findings of medial Parapatellar plicae in subjects who underwent knee arthroscopy.
In our study, $26.9 \%$ of patients whose MRI was negative for plica were found to have one during arthroscopy. It is thus apparent that MRI often misses a significant percentage of synovial plicae in the knee. The excision of plica, after not responding to conservative treatment, yielded great symptom relief [10]. It is important to note that delay in treatment of medial Parapatellar plica syndrome will lead to cumulative chondral damage of the medial femoral condyle $[8,9]$. Arthroscopic debridement of medial Parapatellar plicae may hence play a role in controlling the progression of the mechanical and inflammatory processes that culminate in medial compartment osteoarthritis and subsequent need for arthroplasty.

This study showed a relatively low sensitivity of $73 \%$ and low specificity of $31 \%$ of MRI (Table 3). Another meta-analysis that included seven studies in 2014 reported on the pooled sensitivities of the MRI in detecting a medial Parapatellar plica as $77 \%$ with a $95 \%$ confidence interval of $63 \%$ to $87 \%$ [11]. The same study found a pooled specificity of $58 \%$ (95\% CI: $47 \%-70 \%$ ) for the diagnostic capacity of the MRI. This further supports our findings regarding the poor ability of MRI in detecting medial Parapatellar plicae. We took a closer look and calculated the specificity and sensitivity of MRI of patients when divided into two groups above and below 40 years. A difference was found that suggests that the MRI is more reliable in younger individuals as specificity was $41.7 \%$ and sensitivity $88 \%$ for those 40 
years of age or less and for those older than 40 years specificity was $23.5 \%$ and sensitivity $59.3 \%$.

Several factors could possibly contribute to MRI sub-optimal sensitivity and even lower specificity. Mechanically, flexion and extension of the knee change the apparent dimensions and orientation of plicae. With the knee in 30 to 45 degrees of flexion, a medial Parapatellar plica will be impinging on the medial femoral condyle, while it will not with the knee in full extension [15]. In addition, it is important to note that knee effusions may obscure reading of plicae by MRI, particularly on T2 weighted images, due to their similar appearance [7].

The reliance on MRI for decision making in diagnosing medial Parapatellar plica syndrome and the subsequent need for arthroscopic excision may not be the best strategy. Clinical impression remains a very important factor in the diagnosis and should be considered by third-party payers when deciding on coverage of arthroscopic surgery. Stubbing et al. [11] demonstrated that the sensitivity was increased to $90 \%$ and the specificity to $89 \%$ when combining physical exam and MRI findings as performed by a specialist.

In this study, an experienced musculoskeletal radiologist read and reported the MRIs blindly. However, since it is a retrospective study and featuring a relatively small sample size, selection bias as always is a concern. Additionally, joint effusion may obscure MRI readings because the plica and the effusion appear similar on T2 weighted images. Also to note is the lack of detailed recorded data on patient's signs and symptoms.

Our study suggests that MRI readings are inferior to arthroscopy (the gold standard) in their diagnostic capacity. The only way to truly know if a plica is the culprit behind a patient's knee pain is to resect the plica and observe resolution of the pain [11]. This has implications for future research, stressing the need to define better and less invasive diagnostic techniques and the importance of clinical evaluation on part of the treating surgeon.

\section{Conclusion}

In conclusion, we found that about $25 \%$ of people with a medial Parapatellar plica eluded detection by MRI as read by an expert radiologist. As such we feel it is impractical to base medical coverage for arthroscopic treatment on a modality with $73 \%$ sensitivity. Arthroscopy is currently both the gold standard diagnostic modality and treatment method for medial Parapatellar plica syndrome. Treatment of medial Parapatellar plicae is of concern as it will prevent progression of the disease to medial compartment osteoarthritis of the knee, thereby decreasing the need for arthroplasty and its burden on society.

\section{References}

1. Paradowski PT, Bergman S, Sunden-Lundius A, Lohmander LS, Roos EM (2006) Knee complaints vary with age and gender in the adult population. Population-based reference data for the Knee injury and Osteoarthritis Outcome Score (KOOS). BMC Musculoskelet Disord 7: 38.

2. Jee WH, Choe BY, Kim JM, Song HH, Choi KH (1998) The plica syndrome: diagnostic value of MRI with arthroscopic correlation. J Comput Assist Tomogr 22: 814-818.

3. Kim SJ, Choe WS (1997) Arthroscopic findings of the synovial plicae of the knee. Arthroscopy 13: 33-41.

4. Sakakibara J (1976) Arthroscopic Study on Iino's Band (plica synovialis medio patellaris). J Jap Orthop Ass 50: 513-522.

5. Lyu SR, Lee CC, Hsu CC (2015) Medial Abrasion Syndrome: A Neglected Cause of Knee Pain in Middle and Old Age. Medicine (Baltimore) 94: e736.

6. Ewing JW (1993) Plica: Pathologic or Not? J Am Acad Orthop Surg 1: 117-121.

7. Garcia-Valtuille R, Abascal F, Cerezal L, García-Valtuille A, Pereda T, et al. (2002) Anatomy and MR imaging appearances of synovial plicae of the knee. Radiographics 22: 775-784.

8. Hayashi D, Xu L, Guermazi A, Kwoh CK, Hannon MJ, et al. (2013) Prevalence of MRI-detected mediopatellar plica in subjects with knee pain and the association with MRI-detected patellofemoral cartilage damage and bone marrow lesions: data from the Joints On Glucosamine study. BMC Musculoskelet Disord 14: 292.

9. Kan H, Arai Y, Nakagawa S, Inoue H, Hara K, et al. (2015) Characteristics of medial plica syndrome complicated with cartilage damage. Int Orthop 39: 2489-2494.

10. Prejbeanu R, Poenaru DV, Balanescu AD, Mioc ML (2017) Long term results after arthroscopic resection of medial plicae of the knee-a prospective study. Int Orthop 41: 121-125.

11. Stubbings N, Smith T (2014) Diagnostic test accuracy of clinical and radiological assessments for medial patella plica syndrome: a systematic review and meta-analysis. The Knee 21: 486-490.

12. Vassiou K, Vlychou M, Zibis A, Nikolopoulou A, Fezoulidis I, et al. (2015) Synovial plicae of the knee joint: the role of advanced MRI. Post Grad Med J 91: 35-40.

13. Yang LM (2017). Treatment of medial compartment knee osteoarthritis by arthroscopic 'L' medial release procedure. Int Orthop 41: 2025-2035.

14. Lyu SR, Chiang JK, Tseng CE (2010) Medial plica in patients with knee osteoarthritis: a histomorphological study. Knee Surg Sports Traumatol Arthrosc 18: 769-776.

15. Lyu SR (2007) Relationship of medial plica and medial femoral condyle during flexion. Clin Biomech 22: 1013-1016. 